\title{
Context-Aware Computing and Nature-Inspired Computing
}

\section{ICCASA and ICTCC 2019}

\author{
Phan Cong Vinh ${ }^{1}$
}

Published online: 26 April 2020

(C) Springer Science+Business Media, LLC, part of Springer Nature 2020

\begin{abstract}
The part of this special issue with the title of "Context-aware Computing and Nature-inspired Computing: ICCASA and ICTCC 2019" edited by Prof. Phan Cong Vinh is presented to scientists, researchers, experts and students in the fields of context-aware computing and nature-inspired computing. Hopefully, they will find this part stimulating their research related to the hot topics of contextual cognitive computing and nature-inspired computing and being useful for their future work.
\end{abstract}

Keywords Context-aware computing · Context-aware models · Context-awareness-based systems ·

Nature-inspired computing

\section{Introduction}

On behalf of the Editorial Board, we welcome you on the part of the "Mobile Networks and Applications" journal. In this part, we present six extended papers selected from contributions at $8^{\text {th }}$ EAI International Conference on Context-Aware Systems and Applications (ICCASA 2019) and $5^{\text {th }}$ EAI International Conference on Nature of Computation and Communication (ICTCC 2019) spanning many different aspects of context-aware computing and nature-inspired computing.

This part, with papers contributed by researchers from academia, will serve as a reference for researchers, scientists, experts and students in computer science and computer engineering, as well as developers and practitioners of designing computer systems and networks by providing them with

The editorial for the part of Special Issue including extended papers selected from contributions at $8^{\text {th }}$ EAI International Conference on Context-Aware Systems and Applications (ICCASA 2019) and $5^{\text {th }}$ EAI International Conference on Nature of Computation and Communication (ICTCC 2019)

Phan Cong Vinh

pcvinh@ntt.edu.vn

1 Faculty of Information Technology, Nguyen Tat Thanh University, 300A Nguyen Tat Thanh Street, Ward 13, District 4, Ho Chi Minh City, Vietnam the cutting-edge research results and future opportunities and trends. These contributions include formal methods and practical applications for context-aware computing and nature-inspired computing. In other words, this part covers emerging research topics related to theory and applications for contextual cognitive computing and nature-inspired computing.

\section{Contents}

The papers in this part reflect some recent advances in context-aware computing and nature-inspired computing. Specifically, this part covers many different topics of contextaware computing and nature-inspired computing as follows:

Paper 1 by Jiaqi Yin, Huibiao Zhu and Phan Cong Vinh [6] presents that as a storage system architecture optimized for Facebook's photo application, Haystack has four main advantages than before, including high throughput and low latency, fault-tolerance, cost-effectiveness and simplicity. With its widespread use, its validity and other major properties abstracted from the architecture need to be analyzed in a formal framework. However, there is nearly no research conducted to describe the communications and properties in Haystack. In this paper, authors focus on the internal design of serving and uploading a photo of Haystack architecture and apply Communicating Sequential Processes (CSP) to formalize them in detail. By feeding the models into the model checker Process Analysis Toolkit (PAT), authors 
have verified some crucial properties, including basic property and supplementary properties. Basic property contains Deadlock Freedom. Supplementary properties include synchronous concurrent access, asynchronous concurrent access, synchronous concurrent access with the same client, synchronous concurrent upload and synchronous concurrent upload with the same client. Finally, according to the verification results, authors believe that from the CSP's perspective, the properties of Haystack architecture is valid, which means that it meets the requirements of the documents of Facebook.

Paper 2 by Hiep Xuan Huynh, Huy Quang Dang, Huong Hoang Luong, Linh My Thi Ong, Nghia Duong-Trung, Toan Phung Huynh, Van-Huy Pham and Bernard Pottier [2] reports that the brown plant hopper $(\mathrm{BPH})$ is a crucial pest of rice in tropical zones like the Mekong Delta of Vietnam. It economically causes severe loss to the rice harvest via direct nutritional depletion. Many studies address the BPH surveillance by using networks of wireless sensors that are mounted on light traps. However, these approaches have not been confirmed as effective deployment due to inoperative light traps' locations. The problem is that the geographical area of towns is not identical, leading to unnecessary redundancy of sensors and light traps. The aim in this paper is to optimize the locations of BPH sensor networks by utilizing cellular automata and honeycomb architecture which have not been affected by the spatial characteristic geographically. The authors have made several contributions regarding the mentioned problem by (i) quantitatively proving that the deployment cost of $\mathrm{BPH}$ sensor networks is significantly reduced, and consequently (ii) optimizing the BPH sensor network. Therefore, the appropriate configuration of the network is maintained in any circumstances. The experiments have been performed on BPH surveillance networks in Hau Giang, a substantial rice province in the Mekong Delta of Vietnam.

Paper 3 by Iqbal H. Sarker, Alan Colman, Jun Han, Asif Irshad Khan, Yoosef B. Abushark and Khaled Salah [4] formulates the problem of building a contextaware predictive model based on user diverse behavioral activities with smartphones. In the area of machine learning and data science, a tree-like model as that of decision tree is considered as one of the most popular classification techniques, which can be used to build a data-driven predictive model. The traditional decision tree model typically creates a number of leaf nodes as decision nodes that represent context-specific rigid decisions, and consequently may cause overfitting problem in behavior modeling. However, in many practical scenarios within the context-aware environment, the generalized outcomes could play an important role to effectively capture user behavior. In this paper, authors propose a behavioral decision tree, "BehavDT" context-aware model that takes into account user behavior-oriented generalization according to individual preference level. The BehavDT model outputs not only the generalized decisions but also the context-specific decisions in relevant exceptional cases. The effectiveness of BehavDT model is studied by conducting experiments on individual user real smartphone datasets. The experimental results show that the proposed BehavDT context-aware model is more effective when compared with the traditional machine learning approaches, in predicting user diverse behaviors considering multidimensional contexts.

Paper 4 by Yuan Fei, Huibiao Zhu and Phan Cong Vinh [1] reports that Named Data Networking (NDN) is a new promising architecture of information-centric networking. Traditional mechanisms of access control can no longer fit appropriately, because of its caching property. In this paper, authors make the very first attempt to formally analyze this access control solution. Inspired by the basic BAN logic, authors add some new notions to it to propose the BAN-like logic. It can describe the access control solution and the procedures of the access control solution are idealized. Under several security goals with a set of logical postulates, the idealized procedures are analyzed. Authors give the modification to make the solution safer. Then authors introduce the man-in-themiddle attack into the access control solution and check the insecurity properties. The improvement is given according to the result. The study helps to improve security and protect against various attacks for the access control solution.

Paper 5 by Phuong L. Vo, Tuan-Anh Le and Nguyen H. Tran [5] presents that many rate allocation algorithms for multipath flows which satisfy max-min fairness are centralized and not scalable. Upward max-min fairness is a well-known relaxation of max-min fairness and can be achieved by an algorithm extended from water-filling algorithm. In this paper, authors propose a price-based multipath congestion control protocol whose equilibrium point satisfies upward max-min fairness. The protocol is derived from a network utility maximization model for multipath flows.

Paper 6 by Duc-Minh Ngo, Cuong Pham-Quoc and Tran Ngoc Thinh [3] reports that Software-Defined Networking (SDN) has became one of the most efficient network architectures to deal with complexity, policy control improvement, and vendor dependencies removal. Besides, with the diversity of network attacks, the SDN architecture faces many security issues that need to be taken into account. In this work, authors propose an architecture for SDNbased secured forwarding devices (switches) by extending the previous architecture - HPOFS (High-Performance secured OpenFlow Switch) with multiple security functions including lightweight DDoS (Distributed Denial of Service) mechanisms, signature-based and anomaly-based 
IDS (Intrusion Detection System). Authors implement the architecture on a heterogeneous system including host processors, GPU (Graphics Processing Unit), and FPGA (Field Programmable Gate Array) boards. This is the first forwarding device for SDN implemented on a heterogeneous system in the literature. The system not only is enhanced security but also provides a high-speed switching capacity based on the OpenFlow standard.

\section{Noteworthy aspects}

This part has the following noteworthy aspects:

- Presenting advances of context-aware computing and nature-inspired computing.

- Formally specifying, developing and verifying contextawareness-based systems.

Therefore, the part can be used as an additional source for graduate courses to learn formal methods in computing. Additionally, it will be worthful to professionals from both academia and industry and often serve the immediate appeal to those who want to contribute to formal methods in computing.

\section{Conclusions}

In preparation for this part, we acknowledge the study of all the authors for their worthful contributions to the part and their excellent efforts, and also reviewers to ensure the high quality of the work presented here. All are extremely professional and cooperative. We would like to thank the Editor-in-Chief, Professor Imrich Chlamtac, for his critical support of the part's assembly process.

\section{References}

1. Fei Y, Zhu H, Vinh PC (2020) Security analysis of the access control solution of ndn using ban logic. Mobile Networks and Applications. https://doi.org/10.1007/s11036-019-01435-z

2. Huynh HX, Dang HQ, Luong HH, Ong LMT, Duong-Trung N, Huynh TP, Pham VH, Pottier B (2020) Bph sensor network optimization based on cellular automata and honeycomb structure. Mobile Networks and Applications. https://doi.org/10.1007/s11036-019-01434-0

3. Ngo DM, Pham-Quoc C, Thinh TN (2020) Heterogeneous hardware-based network intrusion detection system with multiple approaches for sdn. Mobile Networks and Applications. https://doi.org/10.1007/s11036-019-01437-x

4. Sarker IH, Colman A, Han J, Khan AI, Abushark YB, Salah K (2020) Behavdt: a behavioral decision tree learning to build user-centric context-aware predictive model. Mobile Networks and Applications. https://doi.org/10.1007/s11036-019-01443-z

5. Vo PL, Le TA, Tran NH (2020) An upward max-min fairness multipath flow control. Mobile Networks and Applications. https://doi.org/10.1007/s11036-019-01436-y

6. Yin J, Zhu H, Vinh PC (2020) Formalization and analysis of haystack architecture from process algebra perspective. Mobile Networks and Applications. https://doi.org/10.1007/s11036-019-01433-1

Publisher's Note Springer Nature remains neutral with regard to jurisdictional claims in published maps and institutional affiliations. 